showed switching and simultaneous treatment from traditional, spiritual and modern healers. Moreover refusal to hospital admission due to limited decision making or due to self treatment at home, delays in time to reach health facility, not boiling drinking water due to lack of awareness, inadequate knowledge and misconceptions regarding ORS, use of public sources of drinking water and presence of blood in stools were other factors found in our triangulated results.

\section{P2-492 PREDICTORS OF MALARIA IN INDIVIDUALS AGED 15 YEARS AND OVER IN INDONESIA, 2010}

doi:10.1136/jech.2011.142976m.19

${ }^{1} \mathrm{~T}$ Zulfikar, ${ }^{2} \mathrm{M}$ Sudaryo. ${ }^{1}$ Port Health of Makassar, CDC - Ministry of Health, Makassar, South Sulawesi, Indonesia; ${ }^{2}$ University of Indonesia, Jakarta, Indonesia

Introduction Malaria is global health problem. Ii is endemic in 10 of the 11 member countries of from the WHO South East-Asian Region, including Indonesia. In 2008 there were 2.4 million laboratory confirmed cases and 40000 deaths from malaria in Indonesia. Based on the Basic Health Census, the national incidence of malaria in 2010 was 22.9 per 1000 population. This study aimed to describe the predictors of malaria occurrence in individuals aged 15 years and over. Methods Data from the Basic Health Census for 177920 respondents were analysed using cross-sectional methodology.

Results Multivariate analyses found that age, gender, home wall type, region type, home proximity to rivers, forests or beaches, residence in a high-human density area, proximity to farms or plantations, use of bed nets, use of repellent and chemo-prophylaxis predicted malaria occurrence.

Conclusions The findings from this study support the use of repellent by persons living in high risk areas, and argue for the Ministry of Health to improve coverage of malariologist in rural areas and enhance vector control. Further studies using a case-control design are needed to assess the association and interplay between the various factors.

\section{NEGLECTED CONDITIONS}

\section{P2-493 COMPUTER VISION SYNDROME: THE ASSOCIATION WITH ERGONOMIC FACTORS}

doi:10.1136/jech.2011.142976m.20

\section{Z A Rahman,* S Sanip. Universiti Sains Islam Malaysia, Kuala Lumpur, Malaysia}

Introduction The "Computer Vision Syndrome" (CVS) is one of the health effect related to the activities while on computer work. This problem is commonly overlooked and neglected therefore this study was aimed to explore ergonomic risk factors that may contribute to CVS.

Methods Using a cross-sectional study, university staff (academician and support staff) that used computer at least $2 \mathrm{~h}$ per day at work was interviewed using guided questionnaire to get information on sociodemographic, eye symptoms and possible contributing factors. Respondent's workstations were assessed for ergonomic factors. Respondents were considered as having CVS if they reported at least one of the vision symptoms as in the questionnaire. Analysis was using SPSS version 15.0.

Results From 436 respondents, $68.1 \%$ of them reported Computer Vision Syndrome (CVS). $\chi^{2}$ Test showed that CVS was significant with gender, education, duration of computer usage, position of monitor to user, computer screen glare and computer monitor level. Exploring the OR, significantly higher odds for CVS were found among respondents who used computer more than five hours per day (OR: 1.8, CI 1.2 to 2.3), not facing their computer screen while computing (OR: 2.9, CI 1.9 to 4.4), computer screen glaring (OR 2.7, CI 1.4 to 5.1) and high monitor level (OR: 1.5, CI 1.0 to 2.4).

Conclusions Prevalence of computer vision syndrome was high (two in every three). Using computer more than $5 \mathrm{~h}$ per day, not facing computer screen while computing, screen glaring and high monitor level may predispose someone to get CVS.

\section{P2-494 DIFFERENTIAL OUTCOME AND TREATMENT RATES FOR PERIPHERAL VASCULAR DISEASE IN THE BLACK, ASIAN AND WHITE BRITISH POPULATION: SECONDARY ANALYSIS OF ENGLISH HOSPITAL DATA 2003-2009}

doi:10.1136/jech.2011.142976m.21

${ }^{1} \mathrm{~N}$ Ahmad, ${ }^{* 1} \mathrm{C}$ Chan, ${ }^{2} \mathrm{G}$ N Thomas, ${ }^{3} \mathrm{P}$ Gill. ${ }^{1}$ Department of Vascular Surgery, Wirral University Hospital NHS Foundation Trust, Wirral, UK; ${ }^{2}$ Department of Public Health, Epidemiology and Biostatistics, University of Birmingham, Birmingham, UK; ${ }^{3}$ Department of Primary Care Sciences, University of Birmingham, Birmingham, UK

Introduction Peripheral vascular disease is under researched with no outcome data for the UK. It has the same underlying pathology and treatment modalities as coronary heart disease and is just as preventable.

Aim To document treatment and outcomes for lower limb peripheral vascular disease in those aged $50-84$ by ethnic group.

Method Secondary analysis of Hospital Episode Statistics (2003-2009) patient data with 77 million admission episodes. Age standardised prevalence data calculated for major leg amputation, endovascular intervention, surgical revascularisation.

Results The prevalence rate, per 100000 in the White British population is approximately double in males compared with females for: major leg amputation $(\mathrm{m}=9, \mathrm{f}=5)$, endovascular intervention $(\mathrm{m}=150, \mathrm{f}=72)$, elective surgical revascularisation $(\mathrm{m}=41, \mathrm{f}=16)$. Proportional rates (White British=100) of amputation were significantly higher in Black men (242; 95\% CI 199 to 286) and women (475; $95 \%$ CI 363 to 587 ) and lower in Asian men (91; 95\% CI 72 to $110)$ and women (66; 95\% CI 41 to 91). Rates for endovascular intervention were lower for both ethnic groups in men (Black 91; 95\% CI 84 to 97: Asian 85; 95\% CI 81 to 90) and Asian women (52; 95\% CI 46 to 58 ) but not Black women (138; 95\% CI 126 to 151). Elective surgical revascularisation rates were also significantly lower in men (Black 74; 95\% CI 63 to 85: Asian 39; 95\% CI 31 to 46) and women (Black 72; 95\% CI 53 to 91: Asian 24; 95\% CI 14 to 34).

Conclusion Blacks have significantly higher rates of leg amputation with significantly lower rates of both endovascular and surgical revascularisation. Asians have significantly lower rates of amputation and revascularisation. These variations warrant further investigation.

\section{P2-495 PREDICTIVE FACTORS FOR NON-REPETITION OF THE TUBERCULIN TEST IN PATIENTS WITH HIV/AIDS: A SURVIVAL ANALYSIS}

doi:10.1136/jech.2011.142976m.22

${ }^{2} \mathrm{~L} C \mathrm{R}$ V Moura, ${ }^{1} \mathrm{M}$ D F P M Albuquerque, ${ }^{*}{ }^{2,3} \mathrm{R}$ Ximenes, ${ }^{2,3} \mathrm{H} \mathrm{R}$ Lacerda, ${ }^{2,3} \mathrm{D}$ M Filho, ${ }^{1} \mathrm{R}$ M S Silva, ${ }^{2} \mathrm{C}$ D P Freitas, ${ }^{4} \mathrm{M}$ R L Byington, ${ }^{5} \mathrm{M}$ T S Barbosa. ${ }^{1}$ Centro de Pesquisas Aggeu Magalhães, Fundação Oswaldo Cruz, Recife, Pernambuco, Brazil; ${ }^{2}$ Departamento de Medicina Tropical, Universidade Federal de Pernambuco, Recife, Pernambuco, Brazil; ${ }^{3}$ Universidade de Pernambuco, UPE, Recife, Pernambuco, Brazil; ${ }^{4}$ Instituto Nacional do Cancer, Rio de Janeiro, Rio de Jeneiro, Brazil; ${ }^{5}$ Departamento de Matemática e Estatística, Universidade Federal do Rio de Janeiro, Rio de Janeiro, Rio de Janeiro, Brazil

Introduction Periodic repetition of the tuberculin skin (TST) test in individuals with HIV/AIDS with an initially non-reactive test should be carried out to identify those who should be treated for latent tuberculosis as recommended by the Brazilian Ministry of Health. 
Methods A cohort of HIV positive patients who had initially tested non-reactive to the TST were followed up for the period between November 2007 and February 2010. The Kaplan-Meier method was used to estimate the probability of not repeating the TST and Cox's Regression analysis used to analyse the factors associated with the time until TST was repeated. Cox's multivariate analysis was stratified according to the hospital the patients were followed, as this variable did not respect the principle of proportionality of risk. Results The probability of not repeating the TT by the end of the follow-up period was $42 \%$. The variables that remained associated with repetition of the TT in the final Cox multivariate model were: aged 40 years or more, BMI between 18.0 and 24.9, being female, and years of schooling.

Conclusion The analysis identified groups of individuals who should be the target of interventions aiming to ensure the TT is repeated following an initial non-reactive test in individuals infected with HIV.

\section{P2-496 AN INTERACTION OF SOCIAL SUPPORT AND REMOTENESS IN THE PREDICTION OF PSYCHOLOGICAL DISTRESS}

\section{doi:10.1136/jech.2011.142976m.23}

J Allen, ${ }^{*}$ K Inder, B Kelly, J Attia, T Lewin. Centre for Brain and Mental Health Research, University of Newcastle, Newcastle, New South Wales, Australia

Introduction The influence of social support on mental health outcomes may vary with remoteness in Australian communities. However, the potential variation of determinants of mental health with remoteness has rarely been directly examined. The current research aims to examine the association of social support and distress with remoteness.

Methods Community cohorts from rural and urban New South Wales were combined ( $N=5924$; mean age $=61.1 \mathrm{yrs}$; $44.2 \%$ Male). Standardised scores were used to facilitate assessment of participant satisfaction with social support networks as measured by the Duke Social Support Index and Interview Schedule for Social Interaction. Remoteness was assessed using a continuous measure, the Accessibility and Remote Index of Australia. The association between demographic characteristics, social support, remoteness, the interaction of social support and remoteness, and psychological distress (cut-off $>24$ on the Kessler 10) was studied using logistic regression. Results Younger age (OR 0.99; 95\% CI 0.98 to 0.99), not being in a married or defacto relationship (OR 1.50; 95\% CI 1.19 to 1.93 ) and decreased social support (OR $0.48 ; 95 \%$ CI 0.45 to 0.53 ), but not gender or remoteness, significantly predicted psychological distress. There was a significant interaction of social support by remoteness (OR 1.12; 95\% CI 1.03 to 1.22 ).

Conclusion Social support displayed a differential effect on distress outcomes across urban and rural environs. These results suggest that remoteness and associated community characteristics may moderate the protective influence of social support and that urban environments may be particularly influenced by very low levels of social support.

\section{P2-497 SELF-REPORT OF ORAL HEALTH IN THE ELDERLY AND ASSOCIATED FACTORS}

doi:10.1136/jech.2011.142976m.24

${ }^{1} \mathrm{D} D$ da Silva, ${ }^{2} \mathrm{R}$ B de Held, ${ }^{2} \mathrm{~S}$ V de Souza Torres, ${ }^{2} \mathrm{M} D \mathrm{D}$ R de Sousa, ${ }^{2} \mathrm{~A} L \mathrm{~L}$ Neri, ${ }^{3} \mathrm{~J}$ L F Antunes. ${ }^{1}$ Faculdade de Odontologia, Universidade de Sao Paulo, Sao Paulo, Sao Paulo, Brazil, '2Universidade Estadual de Campinas, Sao Paulo, Sao Paulo, Brazil; ${ }^{3}$ Faculdade de Saude Publica, Universidade de Sao Paulo, Sao Paulo, Sao Paulo, Brazil

Background The self-report of oral health is an index of subjective functional, social and emotional well-being related to oral status, and is an important determinant of the demand for dental services. This study aimed to assess this outcome and associated factors among the elderly.

Methods A cross-sectional study was conducted with 876 participants in a representative sample of elders (65 years or older) in Campinas, São Paulo, Brazil. Dental examinations followed criteria standardised by the WHO for epidemiological surveys on oral health. The self-report of oral health used the GOHAI-Geriatric Oral Health Assessment Index. Individuals were classified by sociodemographic characteristics, dental status and the prevalence of biological frailty. Data analysis used Poisson regression models, considering sample weights and the complex structure of cluster sampling.

Results Among participants, $18.3 \%$ preserved more than 20 teeth; $38.2 \%$ wore full dentures in both arches, $8.5 \%$ needed a denture in at least one dental arch. The average GOHAI index was high: 33.9 (maximum=36.0). Preserving more than 20 teeth, using denture in both arches, not needing such treatment, presenting with no alteration of oral mucosa and no signs of biological frailty were significantly associated with better self-perceived oral health $(\mathrm{p}<0.05)$.

Conclusions The assessment of self-perceived oral health allowed identifying the main factors that associate with this outcome. This tool may contribute to the planning of dental services, instructing strategies for health promotion aimed at an improved quality of life of people within this age group.

\section{P2-498 PARENTS' PERCEPTIONS AND PRACTICES REGARDING CONSANGUINITY RELATED TO $\beta$ - THALASSAEMIA: A MATCHED CASE CONTROL STUDY}

doi:10.1136/jech.2011.142976m.25

M Beg,* R Siddiqui, K Ahmad, K Humayun. Aga Khan University Hospital, Karachi, Pakistan

Introduction $\beta$-thalassaemia is most common genetically transmitted disorder in Eastern Mediterranean region including Pakistan. Like other recessive disorders, prevalence increases through consanguinity; Pakistan Demographic and Health Survey reports $50 \%$ consanguineous marriages. Perceptions about consanguinity in our population have recently not been studied. Objectives were to determine consanguinity related characteristics among parents of children with thalassaemia and without thalassaemia visiting a major health centre in Karachi-Pakistan. To compare consanguinity practices among such families and to assess susceptibility, benefits and barriers towards thalassaemia.

Methods Sample of 340 cases and 340 controls; age-sex matched were selected from February to April 2010. Parents of thalassaemic children and parents of non-thalassaemic children were interviewed after consent. Consanguinity related characteristics followed in families were inquired. Perceptions regarding susceptibility to thalassaemia, benefits and barriers to screening were determined. Conditional logistic regression used to calculate matched OR. Parents' approaches towards index child were assessed.

Results Compared to no cousin marriage, parents of thalassaemic children had more cousin marriages (MOR: 7.3, 95\% CI 3.6 to 14.6), and grand-parents cousin marriage (MOR: 2.8 , 95\% CI 1.8 to 4.4 ) than controls, more cousin marriages occurred in immediate family in last 5 years of cases than controls (MOR: 2.7, 95\% CI 1.7 to 4.3 ) adjusting for ethnicity defined by mother tongue. Among case parents, (51\%) were taking index child to social events and only $21 \%$ were satisfied with attitudes of other's towards index child.

Conclusion Study supports hypothesis that among parents of thalassaemic (cases), practices and perceptions favouring consanguinity were greater as compared to parents of non-thalassaemic children (controls). 